\title{
Evaluation of linear losses in ventilation ducts with a rounded rectangle cross-section
}

\author{
Kazimierz Peszynski ${ }^{1,{ }^{*}}$, Václav Tesar̆ ${ }^{2}$ and Ewa Kuliś ${ }^{1}$ \\ ${ }^{1}$ UTP University of Science and Technology, Al. Prof. S. Kaliskiego 7, Bydgoszcz, Poland \\ ${ }^{2}$ Václav Tesař, Institute of Thermomechanics of the CAS, v. v. i., Dolejškova 1402/5, 18200 Praha 8, \\ Czech Republic
}

\begin{abstract}
The paper presents a new ventilation system characterized by the cross-section of the channels in the shape of a rounded rectangle. The basic research problem was the determination of local losses in the system components. To achieve this goal, it is necessary to know the linear losses in the ducts. The principle of determining both types of losses was presented. The friction coefficients of linear pressure losses referred to the unit of the straight length of the duct section $\zeta_{l}\left[\mathrm{~m}^{-1}\right]$ as well as generalized dimensionless friction coefficient of linear pressure losses $\lambda[-]$ is presented. The main achievement of the paper is the statement that the lambda coefficient $\lambda$ can replace the coefficients $\zeta_{l}$ for all 79 examined duct cross-sections.
\end{abstract}

\section{Introduction}

In ventilation systems, as in air conditioning, the basic sections are rectangular and circular sections. Both types have their advantages and disadvantages. In this work, rectangular ducts with rounded corners are analysed, this cross-section is to combine the advantages of both of the above-mentioned shapes of ventilation ducts.

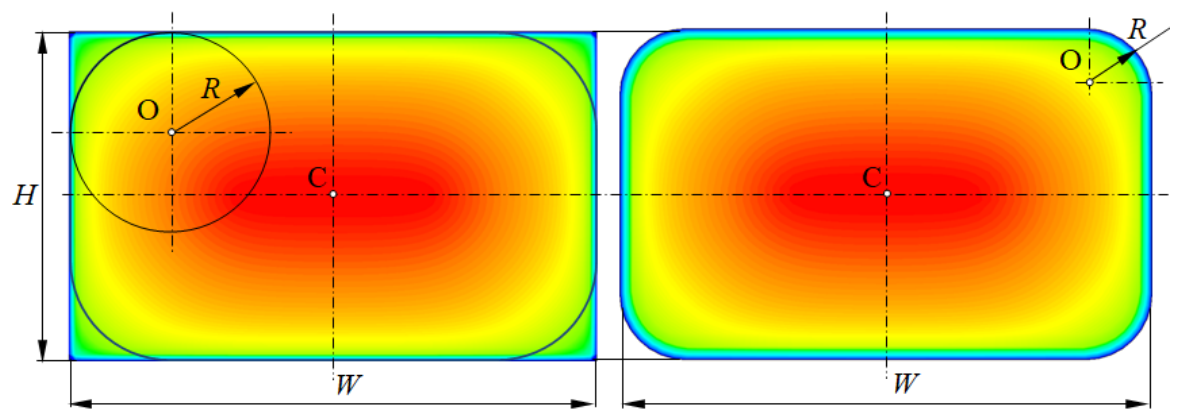

Fig. 1. Genesis of the cross-section: a) initial rectangular b) final rounded rectangular corners.

Figure 1 shows the distribution of air velocity in a rectangular section with dimensions $W \times H=0,8 \mathrm{~m} \times 0,5 \mathrm{~m}$ obtained during simulation in the ANSYS-FLUENT software code, where the average velocity $v_{\text {avg_rec }}=5 \mathrm{~m} \cdot \mathrm{s}^{-1}$ in the duct cross-section was entered at the entrance to the duct. The maximum velocity was observed at the point of intersection of the axis of symmetry $\mathrm{C}$, it was $v_{\max }=v_{\mathrm{Crec}}=6,186 \mathrm{~m} \cdot \mathrm{s}^{-1}$. For the same rectangle, but with corners rounded with a radius $R=0,1 \mathrm{~m}$, was obtained $v_{\max }=v_{\mathrm{Crr}}=6,154 \mathrm{~m} \cdot \mathrm{s}^{-1}$. This is

\footnotetext{
${ }^{*}$ Corresponding author: kazimierz.peszynski@utp.edu.pl
} 
not a surprising result, because it was expected at the same average velocity This result obtained by numerical simulation indicates more even section filling at rounded corners.

\section{Basic parameters of the examined channels}

In Figure 2 selected characteristic cross-sections of the studied channels are presented. The dimensions of the channels are given in meters, e.g. $2.0 \times 1.2$ means $2.0 \mathrm{~m} \times 1.2 \mathrm{~m}$. The coefficient before $A_{\text {rec }}=W \cdot H$ means the indicator of the lost surface of the rectangle as a result of rounding its corners. This coefficient was determined from the formula $A_{\text {rr }} / A_{\text {rec }}$.

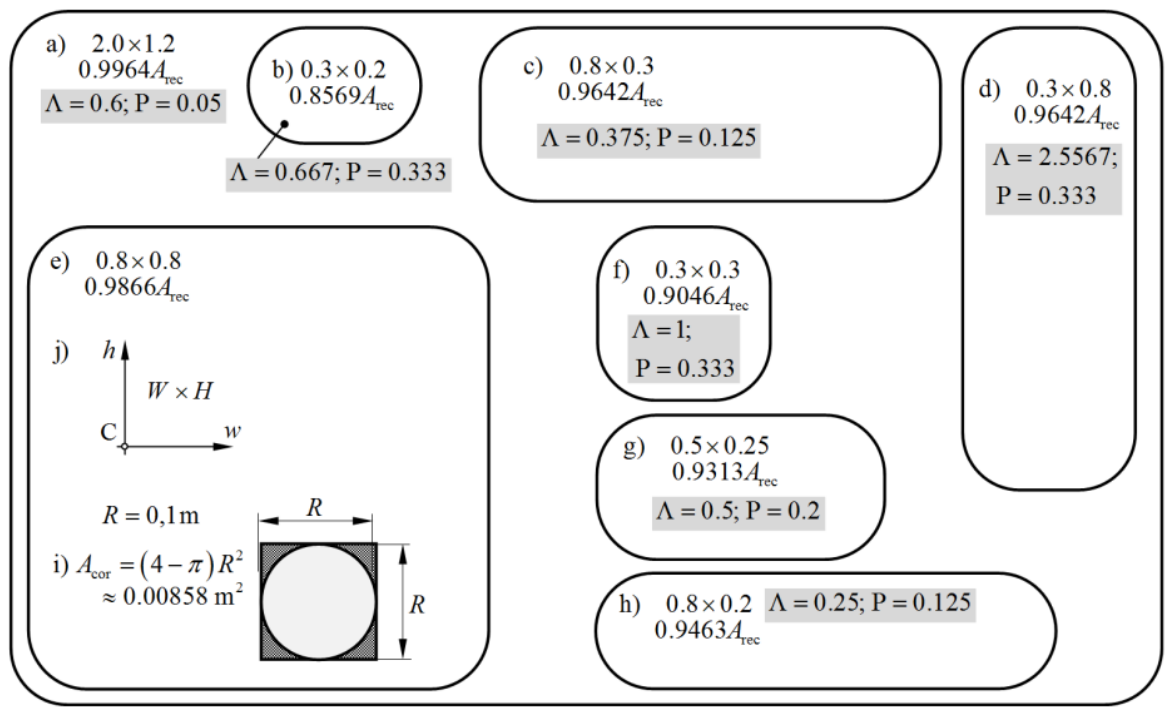

Fig 2. Selected ventilation ducts: a) maximum cross-section, b) minimum cross-section, c), d) maximum cross-section adjustable horizontally and vertically, e) maximum square cross-section, f) minimum square cross-section, g) cross-section for preliminary tests, $h$ ) the most flattened maximum cross-section, i) determination of the lost area rectangle as a result of rounding the cross-section, j) coordinate system.

Since, for technological reasons, the corner rounding radius was the same and was $R=0.1 \mathrm{~m}$ (Fig. 2i), a constant value $A_{\text {cor }}$ was subtracted from the area $A_{\text {rec }}=W \cdot H$, therefore $A_{\mathrm{rr}}=A_{\text {rec }}-A_{\text {cor }}$.

The basic geometrical data of the tested duct cross-section and relative parameters are shown in Figure 3. The relative introduced parameters $\Lambda$ and $\mathrm{P}$ allow the generalization of test results, in accordance with the general trend of entering dimensionless numbers.

The cross-sectional area of the rounded rectangle was determined from the formula:

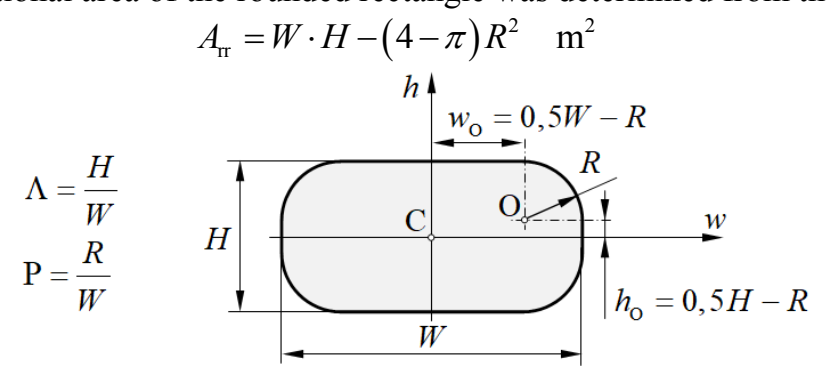

Fig. 3. Basic geometrical parameters of the examined cross-section. 
The cross-sectional area can also be represented in a parametric form $A_{\mathrm{rr}}=f(\Lambda, \mathrm{P}, W)$, thus determination of area $A_{\mathrm{rr}}$ is important when generalizing the results. The parametric form of the surface area was obtained after substituting the parameters specified in the Figure 3 into the equation (1):

$$
A_{\mathrm{rr}}=\left(\Lambda-(4-\pi) \mathrm{P}^{2}\right) W^{2} \quad \mathrm{~m}^{2}
$$

The expression in outer round brackets in the equation (2) is dimensionless and can be treated as a parameter $k_{\mathrm{A}}=\Lambda-(4-\pi) \mathrm{P}^{2}$ characterizing the channel cross-section depending on the channel width $W^{2}$. The factor $k_{\mathrm{A}}$ numerically determines how much the cross-section of the examined duct is smaller than the cross-section of the square with side $W$.

Table 1 presents a list of all cross-sections of the ducts new system under examination. The numerical values in Table 1 were determined on the basis of formula (1), for $R=0,1 \mathrm{~m}$.

Table 1. List of 79 cross-section area of the examined new system.

\begin{tabular}{|c|c|c|c|c|c|c|c|c|c|c|c|c|}
\hline \multirow{2}{*}{\multicolumn{2}{|c|}{$A_{\mathrm{r}}\left[\mathrm{m}^{2}\right]$}} & \multicolumn{11}{|c|}{$W[\mathrm{~m}]$} \\
\hline & & 0,30 & 0,40 & 0,50 & 0,60 & 0,80 & 1,00 & 1,20 & 1,40 & 1,60 & 1,80 & 2,00 \\
\hline \multirow{9}{*}{$H[\mathrm{~m}]$} & 0,20 & 0,051 & 0,071 & 0,091 & 0,111 & 0,151 & & & & & & \\
\hline & 0,25 & 0,066 & 0,091 & 0,116 & 0,141 & 0,191 & 0,241 & & & & & \\
\hline & 0,30 & 0,081 & 0,111 & 0,141 & 0,171 & 0,231 & 0,291 & 0,351 & & & & \\
\hline & 0,40 & 0,111 & 0,151 & 0,191 & 0,231 & 0,311 & 0,391 & 0,471 & 0,551 & & & \\
\hline & 0,50 & 0,141 & 0,191 & 0,241 & 0,291 & 0,391 & 0,491 & 0,591 & 0,691 & 0,791 & & \\
\hline & 0,60 & 0,171 & 0,231 & 0,291 & 0,351 & 0,471 & 0,591 & 0,711 & 0,831 & 0,951 & 1,071 & 1,191 \\
\hline & 0,80 & 0,231 & 0,311 & 0,391 & 0,471 & 0,631 & 0,791 & 0,951 & 1,111 & 1,271 & 1,431 & 1,591 \\
\hline & 1,00 & 0,291 & 0,391 & 0,491 & 0,591 & 0,791 & 0,991 & 1,191 & 1,391 & 1,591 & 1,791 & 1,991 \\
\hline & 1,20 & 0,351 & 0,471 & 0,591 & 0,711 & 0,951 & 1,191 & 1,431 & 1,671 & 1,911 & 2,151 & 2,391 \\
\hline
\end{tabular}

Hydraulic diameter $D_{\mathrm{H}}$ of rounded rectangular (Fig. 3) was determined from equation:

$$
D_{\mathrm{H}}=\frac{4 A_{\mathrm{rr}}}{U_{\mathrm{rr}}}=\frac{4\left(H \cdot W-4\left(1-\frac{\pi}{4}\right) R^{2}\right)}{2(W+H-(4-\pi) R)}=\frac{2\left(\Lambda-(4-\pi) \mathrm{P}^{2}\right)}{1+\Lambda-(4-\pi) \mathrm{P}} W
$$

where: $A_{\mathrm{rr}}$ is area of rounded rectangular, $U_{\mathrm{rr}}-$ perimeter of rounded rectangular, $\Lambda$ - dimensionless height, $\mathrm{P}$ - dimensionless radius, $W, H, R$ - geometrical parameters of the rounded rectangle.

The right side of the equation (3) is interesting. The fraction before the variable $W$ contains only dimensionless construction parameters: the degree of flattening of the crosssection $\Lambda$ and the degree of rounding of the corners $\mathrm{P}$. This is a number factor which, when multiplied by the section width, gives the hydraulic diameter for this cross-section.

\section{Linear losses}

The main task during the research was to determine local losses for individual system elements. The idea of determining local losses is presented in Figure 4. 
b)

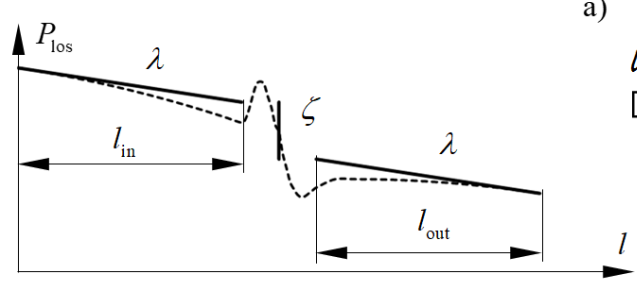

c)

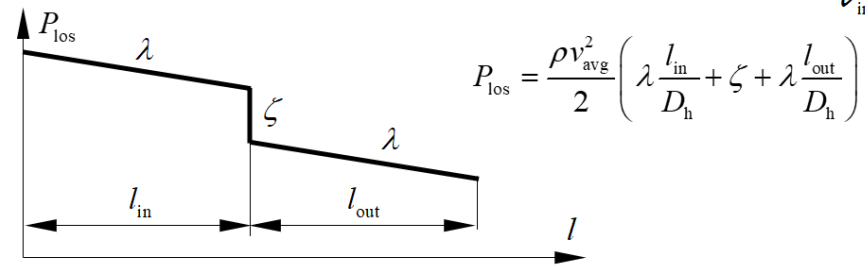

a)

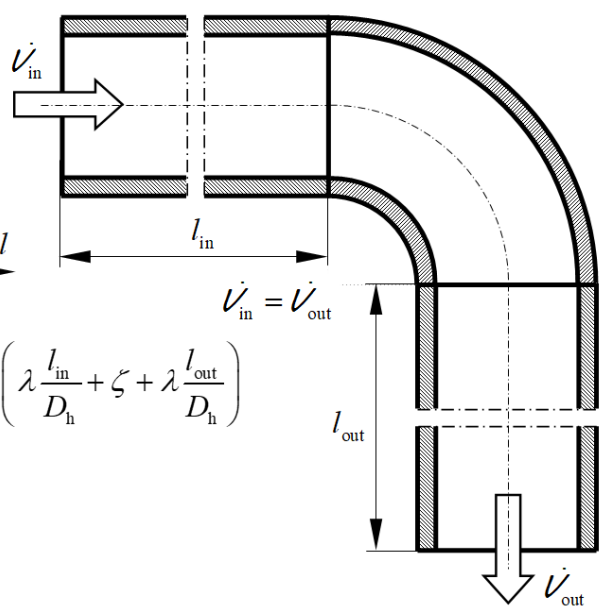

Fig. 4. Basic geometrical parameters of the examined cross-section.

Figure 4 shows a ventilation duct with an arc, at which local losses should be determined $\zeta$. A direct measurement of these losses is not possible. It is necessary to prepare a measuring system containing: an input channel of length $l_{\text {in }}$, the measured element characterized by the sought local losses $\zeta$, and an output channel of length $l_{\text {out }}$. In the literature it is assumed that $l_{\text {in }}>10 D_{\mathrm{h}}$ and $l_{\text {out }}>10 D_{\mathrm{h}}$. The pressure losses in the system are given by the equation

$$
P_{\mathrm{los}}=\frac{\rho v_{\text {avg }}^{2}}{2}\left(\lambda \frac{l_{\text {in }}}{D_{\mathrm{h}}}+\zeta+\lambda \frac{l_{\text {out }}}{D_{\mathrm{h}}}\right)
$$

It is assumed that the component of the ventilation system causes sudden losses, i.e. it has zero length - compare the figures Fig. $4 \mathrm{~b}$ ) and Fig. $4 \mathrm{c}$ ). The average velocity $v_{\text {avg }}$ value in the channel is also necessary, its determination was the basic problem during the tests due to the wide range of measurements. Note that the expression $0,5 \rho v_{\text {avg }}^{2}$ is kinematic pressure. During the research, the effect of air temperature and atmospheric pressure on pressure loss was also analysed. This impact was found to be insignificant. The impact of the average velocity $v_{\text {avg }}$ measurement inaccuracy was much larger. In view of the above, this effect is omitted further in the study. From the formula (5) it is possible to determine the local loss coefficient in the examined component

$$
\zeta=\frac{2 P_{\text {los }}}{\rho v_{\text {avg }}^{2}}-\frac{\lambda}{D_{\mathrm{h}}}\left(l_{\text {in }}+l_{\text {out }}\right)
$$

From formula (6) it follows that in order to determine the local loss coefficient $\zeta$, it is necessary to know the linear loss coefficient $\lambda$.

Initially, attempts were made to determine linear losses theoretically. There are different theoretical ways to determine this coefficient $\lambda$, the most popular is the phenomenological Colebrook-White equation, obviously used for turbulent flow in smooth and rough pipes.

$$
P_{\text {los }}=\frac{\rho v_{\text {avg }}^{2}}{2}\left(\lambda \frac{l_{\text {in }}}{D_{\mathrm{h}}}+\zeta+\lambda \frac{l_{\text {out }}}{D_{\mathrm{h}}}\right)
$$

where: $k$ is roughness of duct, $D_{\mathrm{H}}$ - hydraulic diameter, Re - Reynolds number. 
Equation (6) has an implicit nature, the result can be obtained only by iterative methods, which in the current state of computational technology do not cause any problems. However, when calculating using this formula, it seems that it applies to hydraulically smooth channels only. This, however, cannot be said about the ducts of the system under investigation. These ducts consist of segments of length $l=1.5 \mathrm{~m}$ or $l=1 \mathrm{~m}$. There is a seal between the segments, which, according to observations resulting from the conducted tests, has a much greater influence on the linear losses coefficient $\lambda$ than that occurring in (1), the surface roughness $k$ of the plates from which the ducts were made. In addition, the sealing effect is stochastic. Therefore, during the tests, $\lambda$ was determined experimentally. The principle of this measurement is illustrated in the Figure 5.

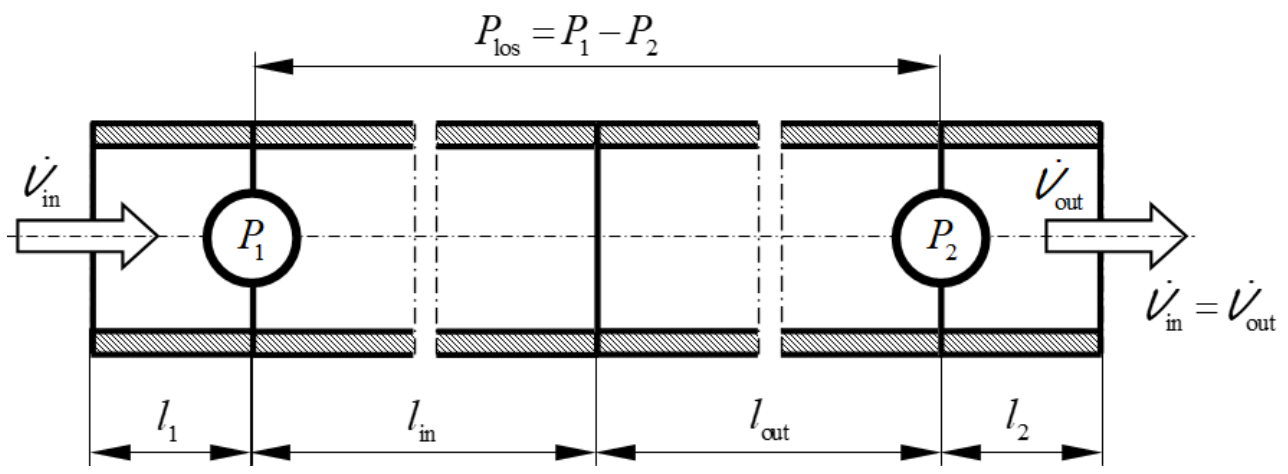

Fig. 5. The idea of measuring the linear loss coefficient $\lambda$.

After removing the tested component (in the situation shown in Fig. 4 segment arc), the inlet and outlet parts are combined into stright one with length $l_{\text {in }}+l_{\text {out }}$. After omitting $\zeta$ in the formula (4) and after its transformation we get the coefficient of local losses

$$
\lambda=\frac{2 P_{\text {los }} D_{\mathrm{H}}}{\rho v_{\text {avg }}^{2}\left(l_{\text {in }}+l_{\text {out }}\right)}
$$

where: $l_{\text {in }}+l_{\text {out }}$ is the length of the straight duct, $P_{\text {los }}$ - pressure drop along the length of the duct, $D_{\mathrm{H}}$ - hydraulic diameter, $\rho$ - air density, $v_{\text {avg }}$ - air average velocity in duct.

The equation (6) illustrates the importance of determining the average flow velocity in the ventilation system duct, therefore the determination of this value publications was devoted to particular attention.

Figure 6 shows the test stand when determining the local loss coefficient for a $60^{\circ}$ segmented elbow for ducts with dimensions $W=0,6 \mathrm{~m}, H=0,2 \mathrm{~m}$. In the upper left corner (Fig. 6a) you can see the straightener of the supply system and the pressure measuring point at the channel inlet. The hydraulic diameter of this channel is $D_{\mathrm{h}}=0,312 \mathrm{~m}$, i.e. the length $l_{\text {in }}>3,12 \mathrm{~m}$, in fact $l_{\text {in }}=6,1 \mathrm{~m}-4$ segments with a length of $1.5 \mathrm{~m}$, and $0.1 \mathrm{~m}$ on the fifth segment. The output channel had the same length, only the fragment with the computer standing on it is visible in the figure. The Fig. 6b) shows a power supply unit whose basic elements are a fan and a spotlight with a chamber. 


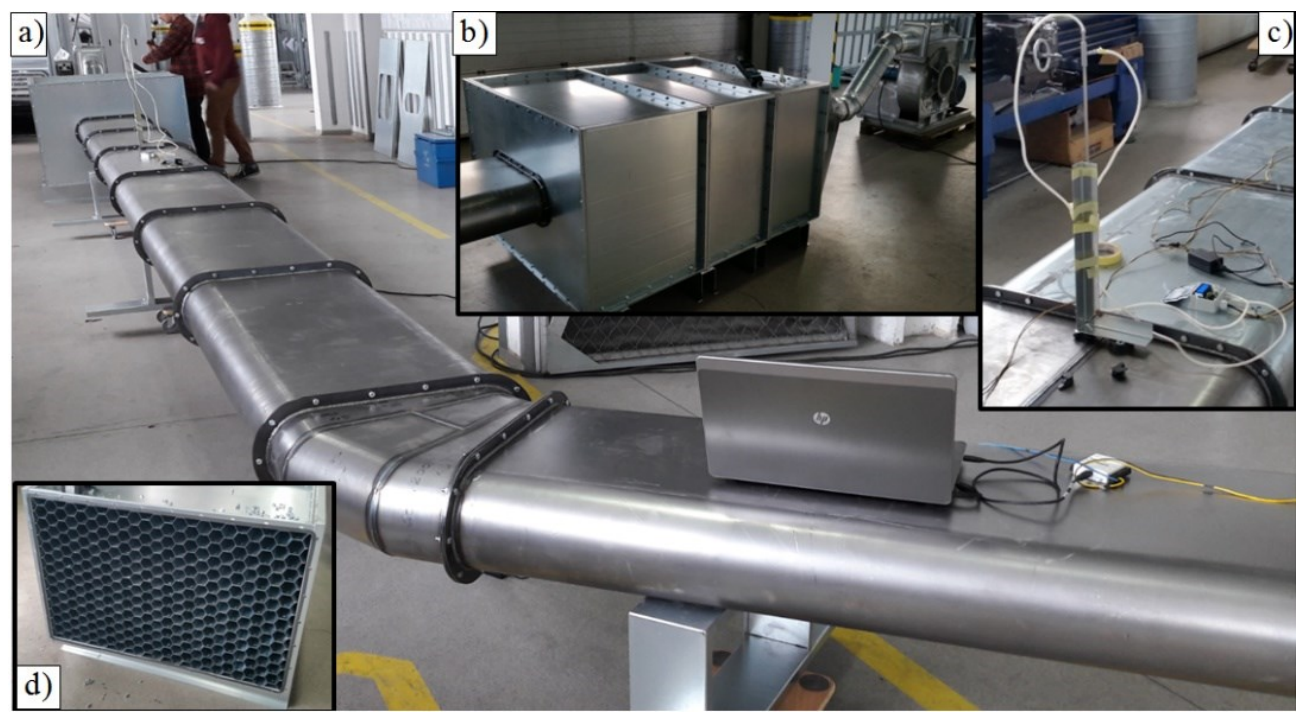

Fig. 6. Test stand before $60^{\circ}$ segmented elbow removal.

Figure $6 \mathrm{c}$ ) shows the pressure measuring point on the axis of the ventilation duct, the basic element is a Prandtl tube. The tube was carefully positioned parallel to the axis of the duct. Fig. 6d) shows the cross-section of the straightener. The straightener channels had the shape of a regular hexagon with a side $a=5 \mathrm{~mm}$, sheet thickness $0.8 \mathrm{~mm}$.

Table 1 shows linear losses marked with the symbol $\zeta_{l}$, for all 79 experimental crosssections tested, given by the formula

$$
\zeta_{l}=\frac{\lambda}{D_{\mathrm{h}}}=\frac{2 P_{\text {los }}}{\rho v_{\text {avg }}^{2}\left(l_{\text {in }}+l_{\text {out }}\right)} \quad\left[\mathrm{m}^{-1}\right]
$$

Table 2. Values of measured linear loss coefficients $\zeta_{l}$ for 79 cross-section area.

\begin{tabular}{|c|c|c|c|c|c|c|c|c|c|c|c|c|}
\hline \multirow{2}{*}{\multicolumn{2}{|c|}{$\zeta_{1}\left[\mathrm{~m}^{-1}\right]$}} & \multicolumn{11}{|c|}{$W[\mathrm{~m}]$} \\
\hline & & 0.3 & 0.4 & 0.5 & 0.6 & 0.8 & 1.0 & 1.2 & 1.4 & 1.6 & 1.8 & 2.0 \\
\hline \multirow{9}{*}{$H[\mathrm{~m}]$} & 0.20 & 0.099 & 0.085 & 0.077 & 0.072 & 0.068 & & & & & & \\
\hline & 0.25 & 0.092 & 0.078 & 0.070 & 0.063 & 0.056 & 0.052 & & & & & \\
\hline & 0.30 & 0.084 & 0.073 & 0.066 & 0.060 & 0.052 & 0.048 & 0.045 & & & & \\
\hline & 0.40 & 0.073 & 0.064 & 0.058 & 0.053 & 0.046 & 0.041 & 0.038 & 0.036 & & & \\
\hline & 0.50 & 0.062 & 0.056 & 0.050 & 0.046 & 0.040 & 0.035 & 0.032 & 0.030 & 0.029 & & \\
\hline & 0.60 & 0.054 & 0.050 & 0.045 & 0.041 & 0.036 & 0.031 & 0.028 & 0.026 & 0.024 & 0.023 & 0.022 \\
\hline & 0.80 & 0.049 & 0.045 & 0.041 & 0.037 & 0.031 & 0.026 & 0.023 & 0.021 & 0.020 & 0.019 & 0.018 \\
\hline & 1.00 & 0.045 & 0.042 & 0.038 & 0.034 & 0.028 & 0.023 & 0.020 & 0.018 & 0.017 & 0.016 & 0.016 \\
\hline & 1.20 & 0.042 & 0.039 & 0.036 & 0.032 & 0.026 & 0.022 & 0.019 & 0.017 & 0.016 & 0.015 & 0.015 \\
\hline
\end{tabular}

This form of linear losses coefficient presentation is very popular in the catalogs of ventilation system published by manufacturers. Graphic form of $\zeta_{l}(w)_{h=c o n s t}$ for selected $h$ is presented also in Fig 8 - dashed lines. Losses in the duct are determined by multiplying the value $\zeta_{l}$ by the length of the duct. Each duct cross-section area has a different linear loss value $\zeta_{l}$. These are the direct results of the experimental studies described above. 


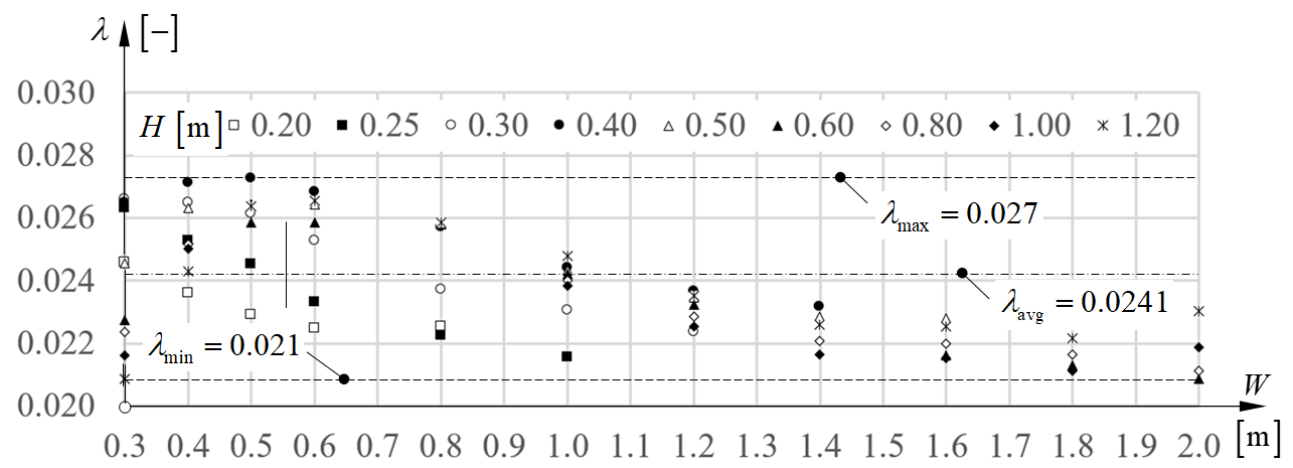

Fig. 7 Dimensionless pressure loss coefficient $\lambda$.

Dimensionless linear loss coefficients $\lambda$ - equation (6) - has a lot more general meaning. In order to obtain a dimensionless pressure loss coefficient $\lambda$ based on the data presented in Table 1, it is necessary to multiply this data by the appropriate hydraulic diameter $D_{\mathrm{H}}$ determined by equation (4). Values of $W$ and $H$ were taken based on the coordinates of the table 2 , the parameter $R=0.1$ was constant for all cross-sections. The result of this multiplication is shown in Fig. 3 in the form of a graph.

The values shown in Figure 7 are within a narrow range of values $\lambda \in(0.020 ; 0.027)$. If we accept as true the sentence included in one of the fundamental textbook on fluid mechanics (Cengel et all, 2006), chap. 8, p. 322 - "An error of 10 percent (or more) in friction factors calculated using the relations in this chapter is the "norm" rather than the "exception." - it is reasonable to calculate the average value for all 79 duct shapes. Therefore

$$
\lambda_{\mathrm{avg}}=\frac{1}{79} \sum_{i=1}^{79} \lambda_{i}=0,024
$$

The value of dimensionless linear loss coefficient $\lambda=\lambda_{\text {avg }}$ determined in the above manner was then used for any comparative analysis of ventilation ducts, first of all for determining local losses coefficient $\zeta$ of system fittings.

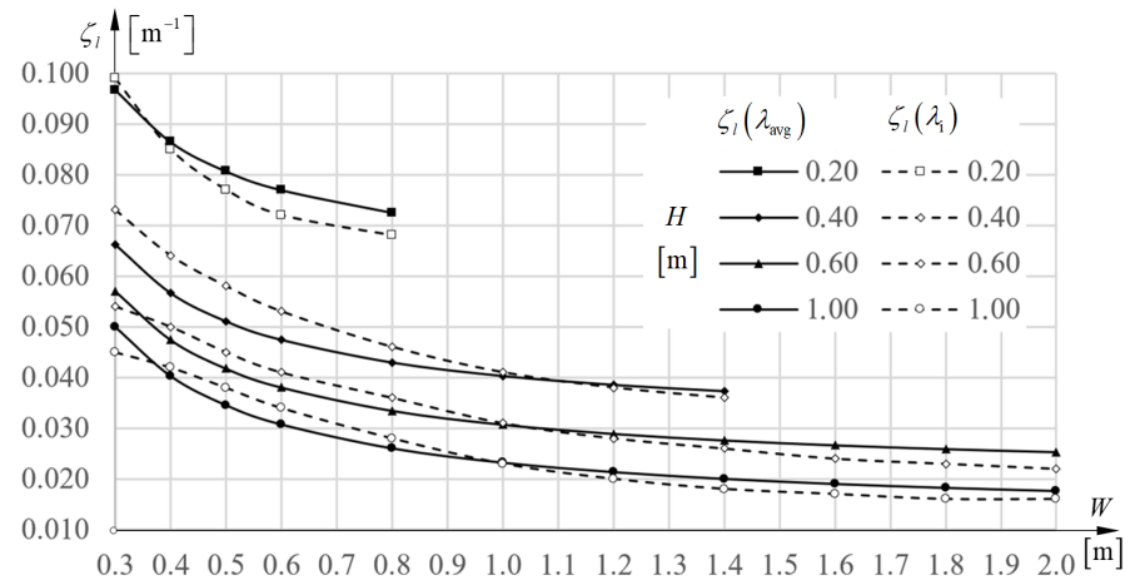

Fig. 8 Comparison of linear loss coefficients $\zeta_{l}$ measured directly and determined on the basis of averaged dimensionless coefficient $\lambda$. 
Figure 8 presents a comparison of $\zeta_{l}$ coefficients measured directly during tests with $\zeta_{l}\left(\lambda_{\text {avg }}, D_{\mathrm{H}}\right)$ coefficients calculated on the basis of average $\lambda_{\text {avg }}$ lambda coefficient and hydraulic diameter $D_{\mathrm{H}}$. For clarity of the image, only 4 sets of results from the available 9 are shown. The calculated coefficients are marked with a solid line and black markers, while the values measured ones with a dashed line and with corresponding white markers. The curves presented in this figure confirm the thesis that all $79 \zeta_{l}$ coefficients can be replaced by one $\lambda$ coefficient.

The reader based on Table 2 and $\lambda=0.024$ can at any time make the missing 5 sets of curves using the left side of the equation (7). This conclusion was very useful during later experimental testing of local pressure losses.

\section{Conclusions}

The dimensionless linear loss coefficient $\lambda[-]$ can replace all dimensional loss coefficients $\zeta_{l}\left[\mathrm{~m}^{-1}\right]$ for 79 examined rounded rectangle duct cross-sections.

The advantage of the above procedure is assimilation experimentally obtained dimensionless linear loss coefficient $\lambda$ to the coefficient determined from the Colebrook White formula (1). In this equation, only the Reynolds number $R e$, the roughness of the plate $k$ and the hydraulic diameter $D_{\mathrm{H}}$ of the cross-section are present, but there is no cross-sectional shape.

The Colebrook-White equation can be applied to a duct with a different cross-section than circular, one should remember about the influence of seals between the duct segments. This goal can be achieved by the correction of the roughness coefficient $k$.

The authors would like to kindly thank the company Nucair Technologies Sp. z o.o., Solec Kujawski, Poland for the performance of tested ducts and delivery of the air supply system. Authors also received institutional support BS 28/2018 granted by Faculty of Mechanical Engineering of UTP University.

\section{References}

1. Y.A, Cengel, J.M. Cimbala: Fluid Mechanics. Fundamentals and Applications, McGraw-Hill, (2006)

2. K. Peszyński (2018) Building a bridge between industry and theory on the example of a new ventilation system. EPJ Web of Conferences Volume 213 (2019)

3. K. Peszyński, J. Novosád, E. Smyk, Ł. Olszewski, P. Dančová (2017) Modelling of air flow rate in significantly flattened rounded rectangular ventilation ducts., EPJ Web of Conferences 180, 02082 (2018)

4. K. Peszyński, L. Olszewski, E. Smyk, and D Perczyński. (2016) Analysis of the Velocity Distribution in Different Types of Ventilation System Ducts, EPJ Web of Conferences 180, 02081 (2018)

5. K. Peszynski, L. Olszewski, E. Smyk, and T. Kasprowicz (2017): Development of New Type Ventilation Ducts System, Proceedings of 23rd International Conference Engineering Mechanics 2017, p. 50 - 53, ISBN 978-80-214-5497-2, ISSN 1805-8248.

6. K. Peszyński, D. Perczyński, E. Smyk, P. Kolber (2017): Experimental Verification of Velocity Distribution in Different Cross-sectional Ventilation Ducts, Proceedings of 23rd International Conference Engineering Mechanics 2017, p. 770 - 783 2017, ISBN 978-80-214-5497-2, ISSN 1805-8248, (2017) 
7. E. Smyk, D. Mrozik, Ł. Olszewski, K. Peszyński (2017) Numerical simulation of minor losses coefficient on the example of elbows, EPJ Web of Conferences 180, 02093 (2018)

8. E. Smyk, P. Mrozik, S. Wawrzyniak, K. Peszyński (2017): Tabular Air Deflector in Ventilation Ducts, Proceedings of 23rd International Conference Engineering Mechanics 2017, p. 882 - 885, ISBN 978-80-214-5497-2, ISSN 1805-8248, (2017) 